\title{
The Primality of $R 1031$
}

\author{
By H. C. Williams* and Harvey Dubner \\ Dedicated to D. H. Lehmer on the occasion of his 80 th birthday
}

\begin{abstract}
A description is given of a technique for proving $R 1031\left(=\left(10^{1031}-1\right) / 9\right)$ a prime.
\end{abstract}
1. Introduction. The repunit numbers, numbers of the form $R_{n}=\left(10^{n}-1\right) / 9$, have been of great interest to mathematical recreationists for many years. One rather peculiar feature of these numbers is the apparent scarcity of primes among them. Indeed, before the writing of this paper only $R 2, R 19, R 23$, and $R 317$ had been identified as primes. In [16] a search for further primes of this form for all $n \leqslant 2000$ turned up only one further candidate for primality, $R 1031$. Unfortunately, the methods of primality testing available at the time were not adequate to prove $R 1031$ a prime. Dubner has since conducted an extended search for repunit primes and, as a result of this work, it is now known that the only possible repunit primes $R_{n}$ for all $n \leqslant 10000$ are the 5 numbers mentioned above. This made the resolution of the question of the primality of $R 1031$ of greater interest.

Since the publication of [16] a great deal of research has been devoted to the problem of primality testing. The very important results of Adelman, Pomerance, and Rumely [1] have been modified by Cohen and Lenstra [5] and have resulted in the tests described by Cohen and Lenstra [6]. It is now possible to test the primality of numbers of up to 200 digits in about 10 minutes of computer time. However, it has been estimated that the application of these rapid, general purpose tests to the problem of determining the primality of a number as large as $R 1031$ might require as many as several hundred hours of computer time.

Since the methods [5], [6] are somewhat complicated to program and would also be rather time-consuming to run on $R 1031$, even on a very fast machine, we decided to make use of the earlier methods of Williams and Judd [13], [14]. The main difficulty in using these techniques is that they are not general purpose methods and require, in order to test a given $N$ for primality, that we first obtain a sufficient number of prime factors from among those which divide, $N \pm 1, N^{2}+1, N^{2} \pm N$ +1 . Also, running the final tests can still be very time-consuming. In this paper we describe how we overcame these difficulties and were able to prove $R 1031$ a prime.

Received December 9, 1985.

1980 Mathematics Subject Classification. Primary 10A25.

* Research supported by NSERC of Canada grant A7649. 
2. The Strategy. Let $N=R 1031$; we have

$$
N-1=10\left(10^{103}+1\right) \cdot \frac{10^{103}-1}{9} \cdot \frac{10^{515}+1}{10^{103}+1} \cdot \frac{10^{515}-1}{10^{103}-1} .
$$

Now $\left(10^{103}-1\right) / 9$ is completely factored in [16] and the complete factorization of $10^{103}+1$ can be obtained from Brillhart et al. [4] and results of Atkin and Rickert mentioned at the end of Williams [12]. Also $\left(10^{515}+1\right) /\left(10^{103}+1\right)$ has $7211 \cdot 9091$ $\cdot 497491 \cdot 569836171 \cdot 2013681931$ as a factor and $T=\left(10^{515}-1\right) /\left(10^{103}-1\right)$ has $41 \cdot 271 \cdot 5905014721$ as a factor (see [16]). With this information we have a completely factored part $F$ of $N-1$, where $F$ is approximately $2.73 \times 10^{251}$. While this represents a very large factor of $N-1$, it does not furnish us with sufficient information for developing a proof that $N$ is a prime.

Dubner and Dubner [7] have developed a special purpose microcomputer that performs multiple-precision operations very quickly; in fact, this very small machine is about as fast as IBM's latest mainframe. This device was used to search for more factors of $N-1$. By utilizing the techniques of Brent and Pollard [2], the factor 326345481191 of $T$ was found and an additional factor 2702394989404991 of $T$ was found by using the $p-1$ method described by Pollard [9]. These factors were determined by the Dubner machine in a matter of a few minutes. With this information we have $N-1=F_{1} C_{1}$, where $F_{1}$, the completely factored part of $N$ - 1 , exceeds $2.40 \times 10^{278}$.

Now the following theorem is well known and true for an arbitrary $N$ (see, for example, Brillhart, Lehmer, and Selfridge [3]).

THEOREM. If for each distinct prime $q_{i}$ which divides $F_{1}$, there exists an integer $a_{i}$ such that

$$
\operatorname{gcd}\left(a_{i}^{(N-1) / q_{1}}-1, N\right)=1
$$

and

$$
a_{i}^{N-1} \equiv 1(\bmod N),
$$

then any prime divisor of $N$ must have the form $m F_{1}+1$.

If $N$ is a prime, the probability that (2.1) does not hold is about $1 / q_{i}$. Thus, we elected to try a single $a$ value such that $(a / N)=-1$ (Jacobi symbol). This means that (2.1) must hold if $q_{i}=2$ and $N$ is a prime. We determine

$$
b \equiv a^{C_{1}}(\bmod N) \text {. }
$$

Let $Q_{i}=F_{1} / q_{i}$, where $q_{i}$ is a prime divisor of $F$. For each distinct $q_{i}$, we then evaluate

$$
c_{i} \equiv b^{Q_{i}}(\bmod N) \text {. }
$$

We see that (2.1) and (2.2) hold if

$$
\operatorname{gcd}\left(c_{i}-1, N\right)=1 \text { and } c_{i}^{q_{1}} \equiv 1(\bmod N) .
$$

Of course, if $\operatorname{gcd}\left(c_{i}-1, N\right) \neq 1$ for some $q_{i}$, we would be compelled to try a different value of $a$ for that particular $q_{i}$.

By using only a single value of $a(=7)$ and our particular $N$ value $(R 1031)$, we were able to run these final tests (for the factors of $F_{1}$ ) and verify in about 18 minutes of AMDAHL $5850 \mathrm{CPU}$ time that if $p$ is a prime factor of $N$, then $p \equiv 1$ 
$\left(\bmod F_{1}\right)$. All programming for the AMDAHL was done in FORTRAN with special assembler language subroutines used for the multiprecise operations of addition, subtraction, multiplication, and division.

It follows from this that if $N$ is the product of three nontrivial factors $f_{1}, f_{2}, f_{3}$, then

$$
N=\left(m_{1} F_{1}+1\right)\left(m_{2} F_{2}+1\right)\left(m_{3} F_{3}+1\right),
$$

where $f_{i}=m_{i} F_{1}+1$ and $m_{i}>0(i=1,2,3$,$) . Hence,$

$$
m_{1}+m_{2}+m_{3} \equiv C_{1}\left(\bmod F_{1}\right) \text {. }
$$

Let $L$ be the remainder on dividing $C_{1}$ by $F_{1}$. Since

$$
m_{1}+m_{2}+m_{3} \geqslant L
$$

we must have $m_{i}>L / 3$ for some $i \in\{1,2,3\}$. Thus, if $N$ is the product of three nontrivial factors, we must have $N>L F_{1}^{3} / 3$. In fact, we found by evaluating $L$ that

$$
L F_{1}^{3} / 3>N
$$

hence $N$ must be the product of at most two prime factors.

Lenstra [8] has shown that if we know that a factor $f$ of $N$ must have the form $f=m s+r$, where $s>N^{1 / 3}$, then a fast algorithm, given in [8], can be used to find $f$. Unfortunately, our value for $F_{1}$ is much less than $N^{1 / 3}$; thus, we decided to search for factors of $N+1, N^{2}+1$, and $N^{2} \pm N+1$. By using trial division, the $p-1$ method, and Pollard's [10] Monte Carlo method, we found

$$
\begin{gathered}
N+1=2^{3} \cdot 3 \cdot 78869803 \cdot C_{2}=F_{2} C_{2}, \\
N^{2}+1=2 \cdot 101 \cdot 2184509 \cdot 134089273 \cdot 1124225381 \cdot C_{4}=2 F_{4} C_{4}, \\
N^{2}+N+1=7 \cdot 13 \cdot 193 \cdot 54223873993 \cdot C_{3}=F_{3} C_{3}, \\
N^{2}-N+1=3 \cdot 37 \cdot 661 \cdot 4236022699 \cdot C_{6}=3 F_{6} C_{6} .
\end{gathered}
$$

Also, $C_{1}, C_{2}, C_{3}, C_{4}, C_{6}$, are composite integers. These new factors were also found in a matter of a few minutes on the Dubner machine. We need

$$
K^{\prime}=\left(F_{1} F_{2} F_{3} F_{4} F_{6}\right) / 2>N^{1 / 3} ;
$$

however, $K^{\prime} \simeq 7.47 \times 10^{341}$ and $N^{1 / 3} \simeq 2.23 \times 10^{343}$; hence, we require one more factor.

After using the Dubner machine to search each of $C_{1}, C_{2}, C_{3}, C_{4}, C_{6}$ for another factor (spending, on the average about 30 hours per $C$ value), we still had found no additional factor. As $C_{6}$ was the last number to be tested and was still in the machine, we simply allowed the machine to keep running on it. After using the $p-1$ method for 110 hours, the factor

$$
\pi=2211993420324463
$$

of $C_{6}$ was discovered. Replacing, $F_{6}$ by $\pi F_{6}$, we now have

$$
K=\left(F_{1} F_{2} F_{3} F_{4} F_{6}\right) / 2>N^{1 / 3} \text {. }
$$

Let $S$ be any factor of $K$ such that $S>N^{1 / 3}$ and let

$$
\bar{F}_{i}=\operatorname{gcd}\left(S, F_{i}\right) \quad(i=1,2,3,4,6) .
$$


Once the final tests for the primes dividing $S$ given in [3], [13], and [14] have been executed (see Section 3 for a full description of these), we know that since $N$ can be the product of at most two primes, we must, if $N$ is composite, have one of 6 possibilities for one of these primes $p<N$. (See Williams and Holte [15].)

By using the Chinese Remainder Theorem, we first solve the system

$$
x \equiv 1\left(\bmod \bar{F}_{1}\right), \quad x \equiv-1\left(\bmod \bar{F}_{2}\right)
$$

for $y$. We then solve each of the 6 systems

(i) $x \equiv y\left(\bmod \bar{F}_{1} \bar{F}_{2}\right), x \equiv N\left(\bmod \bar{F}_{4} \bar{F}_{3} \bar{F}_{6}\right)$ for $r_{1}$;

(ii) $x \equiv y\left(\bmod \bar{F}_{1} \bar{F}_{2}\right), x \equiv-N\left(\bmod \bar{F}_{4}\right), x \equiv N\left(\bmod \bar{F}_{3} \bar{F}_{6}\right)$ for $r_{2}$;

(iii) $x \equiv y\left(\bmod \bar{F}_{1} \bar{F}_{2}\right), x \equiv N\left(\bmod \bar{F}_{4}\right), x \equiv 1\left(\bmod \bar{F}_{3}\right), x \equiv-1\left(\bmod \bar{F}_{6}\right)$ for $r_{3}$

(iv) $x \equiv y\left(\bmod \bar{F}_{1} \bar{F}_{2}\right), x \equiv-N\left(\bmod \bar{F}_{4}\right), x \equiv 1\left(\bmod \bar{F}_{3}\right), x \equiv-1\left(\bmod \bar{F}_{6}\right)$ for $r_{4}$;

(v) $x \equiv y\left(\bmod \bar{F}_{1} \bar{F}_{2}\right), x \equiv N\left(\bmod \bar{F}_{4}\right), x \equiv-N-1\left(\bmod \bar{F}_{3}\right), x \equiv-N+1$ $\left(\bmod \bar{F}_{6}\right)$ for $r_{5}$;

(vi) $x \equiv y\left(\bmod \bar{F}_{1} \bar{F}_{2}\right), x \equiv-N\left(\bmod \bar{F}_{4}\right), x \equiv-N-1\left(\bmod \bar{F}_{3}\right), x \equiv-N+1$ $\left(\bmod \bar{F}_{6}\right)$ for $r_{6}$.

If $N$ is composite, it must have a prime factor $p$ such that $p \equiv r_{i}(\bmod S)$ where $i \in\{1,2,3,4,5,6\}$ and $S>N^{1 / 3}$.

We can now use Lenstra's algorithm to attempt to find $p$. The only difficulty which arises in using this algorithm lies in the determination of whether or not there are integer zeros of a quadratic polynomial $X^{2}-A X+B$ when $A$ and $B$ are large. But, since we usually do not expect to have such integral zeros, the best way to test for this is to find a collection of small primes $t_{1}, t_{2}, t_{3}, \ldots, t_{m}$ such that $t_{i}+S$ (in this case) and determine for each $t_{i}$ its set of $\left(t_{i}-1\right) / 2$ quadratic nonresidues $\left(\bmod t_{i}\right)$. We need, then, only to test whether or not $\Delta=A^{2}-4 B \equiv$ to a quadratic nonresidue of $t_{i}\left(\bmod t_{i}\right)$. Of course, if $\Delta$ is a quadratic residue for each $t_{i}$, then Newton's method can be employed to determine whether or not $\Delta$ is truly a perfect square. We found that with $m=15$ this method worked very well.

We implemented Lenstra's algorithm on the AMDAHL 5850 and tested it thoroughly on a large number of multiprecise composite numbers for which we knew the form of the factors. This algorithm works surprisingly rapidly and when used on the problem of finding a factor of $R 1031$ required only $18 \mathrm{CPU}$ seconds for each of the $6\left(S, r_{i}\right)$ pairs.

3. The Final Tests. It remains to describe the final tests for the factors of $S$. We have already performed these tests for the factors of $\bar{F}_{1}$; hence, we need only perform them for factors of $\bar{F}_{2}, \bar{F}_{3}, \bar{F}_{4}, \bar{F}_{6}$. As was done in [3], we give the final tests for the factors of $\bar{F}_{2}$ in terms of Lucas Functions.

Let $P, Q$ be integers such that $\operatorname{gcd}(P, Q)=1$. Define the Lucas Functions by

$$
V_{n}(P, Q)=\alpha^{n}+\beta^{n}, \quad U_{n}(P, Q)=\left(\alpha^{n}-\beta^{n}\right) /(\alpha-\beta),
$$

where $\alpha, \beta$ are the zeros of $x^{2}-P x+Q$. Put $\Delta=P^{2}-4 Q$.

Final Tests for the Factors of $\bar{F}_{2}$. Let $D$ be a fixed integer such that the Jacobi symbol $(D / N)=-1$. For each distinct prime $q_{i}$ such that $q_{i} \mid \bar{F}_{2}$, find a pair $P, Q$ such that $D=P^{2}-4 Q$,

$$
\operatorname{gcd}\left(N, U_{(N+1) / q_{i}}(P, Q)\right)=1
$$


and

$$
N \mid U_{N+1}(P, Q) \text {. }
$$

In [3] it is shown how $U_{m}(P, Q)(\bmod N)$ can be computed rapidly, even when $m$ is large. However, when $N$ is very large, these final tests as stated here may consume more computer time than necessary. We describe below another means by which these tests can be conducted.

We first note that from the definition of the Lucas Functions we can show that**

$$
\begin{aligned}
& V_{2 m}=V_{m}^{2}-2 Q^{m}, \\
& V_{4 n+2}=V_{2 n} V_{2 n+2}-Q^{2 n} V_{2}, \\
& V_{m}^{2}-\Delta U_{m}^{2}=4 Q^{m}, \\
& V_{m}\left(V_{n}, Q^{n}\right)=V_{n m}(P, Q) .
\end{aligned}
$$

If we define $W_{n} \equiv V_{2 n} Q^{-n}(\bmod N)$, we have

$$
W_{1} \equiv P^{2} Q^{-1}-2(\bmod N) .
$$

Also, from (3.1), (3.2), and (3.3) we get

$$
\begin{aligned}
& W_{2 n} \equiv W_{n}^{2}-2(\bmod N), \\
& W_{2 n+1} \equiv W_{n} W_{n+1}-W_{1}(\bmod N), \\
& \Delta\left(U_{2 n} Q^{-n}\right)^{2} \equiv W_{n}^{2}-4(\bmod N) .
\end{aligned}
$$

Since $W_{0}=2$, we see from (3.5) and (3.6) that the value of $W_{n}$ depends only on that of $W_{1}$; hence, we may regard $W_{n}$ to be a function of $W_{1}$ and $n$ only. When we do this, we can use (3.4) to deduce

$$
W_{n}\left(W_{m}\right) \equiv W_{n m}\left(W_{1}\right)(\bmod N) .
$$

Let $\mathscr{T}_{m}$ denote the pair $\left\{W_{m}, W_{m+1}\right\}$, where each entry of $\mathscr{T}_{m}$ is reduced modulo $N$. Let $m=\left(b_{0}, b_{1}, b_{2}, \ldots, b_{t}\right)_{2}$ be the binary representation of $m$, and define $f_{1}(x)=x^{2}-2, f_{2}(x, y)=x y-W_{1}$. If we also define $c_{0}=b_{0}=1, c_{j+1}=2 c_{j}+$ $b_{j+1}, \mathscr{G}_{i}=\mathscr{T}_{c_{i}}$, then $\mathscr{G}_{t}=\mathscr{T}_{m}$. By (3.6) and (3.7) we can easily deduce that if $\mathscr{G}_{i}=\{x, y\}$, then

$$
\mathscr{G}_{i+1}= \begin{cases}\left\{f_{1}(x), f_{2}(x, y)\right\} & \text { when } b_{i+1}=0, \\ \left\{f_{2}(x, y), f_{1}(y)\right\} & \text { when } b_{i+1}=1 .\end{cases}
$$

Thus, if we are given $W_{1}$, we now have a fast $O(\log m)$ algorithm for computing $W_{m}\left(W_{1}\right) \equiv V_{2 m}(P, Q) Q^{-m}(\bmod N)$, when $W_{1}$ is given by $(3.5)$.

We now give a different formulation of the final tests for the factors of $\bar{F}_{2}$. As before, we let $D$ be a fixed integer such that the Jacobi symbol $(D / N)=-1$. We find $P, Q$ such that $P^{2}-4 Q \equiv D(\bmod N)$ and we determine $W_{1}$ by (3.5). Since $Q$ is usually small, the computation of $Q^{-1}(\bmod N)$ can be done quickly. Compute

$$
W_{1}^{*} \equiv W_{A}\left(W_{1}\right)(\bmod W) \quad\left(A=(N+1) / 2 \bar{F}_{2}\right)
$$

by using the algorithm given above. For each $q_{i} \mid \bar{F}_{2}$, put $Q_{i}=\bar{F}_{2} / q_{i}$,

$$
X_{i} \equiv W_{Q_{i}}\left(W_{1}^{*}\right)(\bmod N) \text {. }
$$

Note that $X_{i} \equiv W_{(N+1) / 2 q_{i}}\left(W_{1}\right)(\bmod N)$ by (3.9). Determine that

$$
\operatorname{gcd}\left(X_{i}^{2}-4, N\right)=1 \text {, }
$$

\footnotetext{
${ }^{* *}$ When there is no doubt as to the arguments $P, Q$ of $V_{n}$ and $U_{n}$, we will omit them.
} 
and for

$$
Y_{i} \equiv W_{q_{i}}\left(X_{i}\right)(\bmod N)
$$

$\left(W_{q_{i}}\left(X_{i}\right) \equiv W_{(N+1) / 2}\left(W_{1}\right)(\bmod N)\right)$, that

$$
N \mid Y_{i}^{2}-4
$$

Since $\operatorname{gcd}(D Q, N)=1$, we see by (3.8) that this formulation of the final tests is correct. If (3.12) should not be true for some prime $q_{i}$, then the tests would have to be repeated with a different $P, Q$ pair, but we would only test those $q_{i}$ 's for which (3.12) failed to be true. Since the probability that (3.12) will not hold when $N$ is a prime is about $1 / q_{i}$, this event is not very likely.

In fact, with $\bar{F}_{2}=F_{2} / 8, D=21, P=5, Q=1$, we found that $N=R 1031$ passed these final tests in about 4 CPU minutes of AMDAHL time.

The ideas developed here for the final tests for the factors of $\bar{F}_{2}$ can be extended to the relevant final tests for the factors of $\bar{F}_{4}, \bar{F}_{3}, \bar{F}_{6}$ given in [13] and [14]. For the generalized Lehmer Functions defined in [11] we now define

$$
W_{j, n} \equiv V_{j, 2 n} Q^{-n}(\bmod N) \text {, }
$$

$\mathscr{T}_{m}=\left\{W_{0, m}, W_{1, m}, W_{0, m+1}, W_{1, m+1}\right\}$ when $k=2$ (used when dealing with the factors of $\left.\bar{F}_{4}\right)$, and $\mathscr{T}_{m}=\left\{W_{0, m}, W_{1, m}, W_{2, m}, W_{0, m+1}, W_{1, m+1}, W_{2, m+1}\right\}$ when $k=3$ (used when dealing with the factors of $\bar{F}_{3}$ or $\bar{F}_{6}$ ). As before, the entries of $\mathscr{T}_{m}$ are reduced modulo $N$; also, for a given $m$, we put $\mathscr{G}_{i}=\mathscr{T}_{c_{i}}$.

If we define

$$
\begin{aligned}
& g_{1}(x, y)=x^{2}-P_{2} y^{2}-2, \quad g_{2}(x, y)=2 x y+P_{1} y^{2}, \\
& g_{3}(x, y, z, w)=x z-P_{2} y w-W_{0,1}, \\
& g_{4}(x, y, z, w)=y z+x w+P_{1} y w-W_{1,1} \\
& =(y+x)(z+w)-x z+\left(P_{1}-1\right) y w-W_{1,1},
\end{aligned}
$$

then when $k=1$, we have $W_{0,0}=2, W_{1,0}=1, W_{0,1} \equiv+P_{2} Q^{-1}-2, W_{1,1} \equiv P_{1} Q^{-1}$ $(\bmod N)$ and

$$
\begin{aligned}
& W_{0,2 n} \equiv g_{1}\left(W_{0, n}, W_{1, n}\right), \quad W_{1,2 n}=g_{2}\left(W_{0, n}, W_{1, n}\right), \\
& W_{0,2 n+1} \equiv g_{3}\left(W_{0, n}, W_{1, n}, W_{0, n+1}, W_{1, n+1}\right), \\
& W_{1,2 n+1} \equiv g_{4}\left(W_{0, n}, W_{1, n}, W_{0, n+1}, W_{1, n+1}\right)(\bmod N) .
\end{aligned}
$$

These forms can be easily verified by using the methods of [11] and the simple results (analogous to (3.1) and (3.2)) that

$$
\begin{aligned}
& v_{2 n}(\rho, Q)=v_{n}(\rho, Q)-2 Q^{n}, \\
& v_{4 n+2}(\rho, Q)=v_{2 n+1}(\rho, Q) v_{2 n}(\rho, Q)-Q^{2 n} v_{2}(\rho, Q) .
\end{aligned}
$$

Thus, if $\mathscr{G}_{i}=\{x, y, z, w\}$, then

$$
\mathscr{G}_{i+1}= \begin{cases}\left\{g_{1}(x, y), g_{2}(x, y), g_{3}(x, y, z, w), g_{4}(x, y, z, w)\right\} & \text { if } b_{i+1}=0 \\ \left\{g_{3}(x, y, z, w), g_{4}(x, y, z, w), g_{1}(z, w), g_{2}(z, w)\right\} & \text { if } b_{i+1}=1\end{cases}
$$

Also, if we write

$$
W_{0, n}=W_{0, n}\left(W_{0,1}, W_{1,1}, P_{1}, P_{2}\right), \quad W_{1, n}=W_{1, n}\left(W_{0,1}, W_{1,1}, P_{1}, P_{2}\right),
$$

then

$$
W_{0, m n} \equiv W_{0, m}\left(W_{0, n}, W_{1, n}, P_{1}, P_{2}\right), \quad W_{1, m n} \equiv W_{1, m}\left(W_{0, n}, W_{1, n}, P_{1}, P_{2}\right)(\bmod N)
$$


By noting that the $U_{m}$ in final test $\alpha$ (the final tests for the factors of $\bar{F}_{4}$ ) of [13] is the same as $V_{1, m}$, we can reformulate test $\alpha$ here in an analogous manner to our reformulation of the final tests for the factors of $\bar{F}_{2}$ given in [3]. We use the previous value of $D$ and select a fixed $C$ value as described in [13]. We then select $H, K$ and compute $P_{1}, P_{2}, Q$ by the formulas given in [13]. We compute $W_{0,1}, W_{1,1}(\bmod N)$. The test has the same structure of that for the factors of $\bar{F}_{2}$, but we replace (3.10) by

$$
\begin{aligned}
& W_{0,1}^{*} \equiv W_{0, A}\left(W_{0,1}, W_{1,1}, P_{1}, P_{2}\right)(\bmod N) \quad\left(A=\left(N^{2}+1\right) / 2 \bar{F}_{4}\right), \\
& W_{1,1}^{*} \equiv W_{1, A}\left(W_{0,1}, W_{1,1}, P_{1}, P_{2}\right)(\bmod N),
\end{aligned}
$$

(3.11) by

$$
\begin{aligned}
& X_{0, i} \equiv W_{0, Q_{i}}\left(W_{0,1}^{*}, W_{1,1}^{*}, P_{1}, P_{2}\right)(\bmod N), \\
& X_{1, i} \equiv W_{1, Q_{i}}\left(W_{0,1}^{*}, W_{1,1}^{*}, P_{1}, P_{2}\right)(\bmod N),
\end{aligned}
$$

(3.12) by

$\operatorname{gcd}\left(X_{1, i}, N\right)=1$ (if this does not occur, we must change the $(H, K)$ pair for $q_{i}$ ),

(3.13) by

$$
Y_{1, i} \equiv W_{1, q_{i}}\left(X_{0, i}, X_{1, i}, P_{1}, P_{2}\right)(\bmod N)
$$

and (3.14) by

$$
N \mid Y_{1, i}
$$

Here, of course, $q_{i} \mid \bar{F}_{4}$ and $Q_{i}=\bar{F}_{4} / q_{i}$.

Using this reformulation of test $\alpha$ with $\bar{F}_{4}=F_{4}$ and $D=21, C=29, H=1$, $K=0$, we found that $N$ passed these final tests in about 16 minutes of AMDAHL CPU time.

If, when $k=3$, we further define

$$
\begin{aligned}
h_{1}(x, y, z)=x^{2}+ & 2 P_{3} y z+P_{1} P_{2} z^{2}-2, \\
h_{2}(x, y, z)=2 x y- & 2 P_{2} y z+\left(P_{3}-P_{1} P_{2}\right) z^{2} \\
h_{3}(x, y, z)=y^{2}+ & 2 z x+2 P_{1} y z+\left(P_{1}^{2}-P_{2}\right) z^{2} \\
h_{4}(x, y, z, u, v, w)= & x u+P_{3}(y+z)(v+w)+\left(P_{1}-P_{2} P_{3}\right) z w \\
& -P_{3} y v-W_{0,1}, \\
h_{5}(x, y, z, u, v, w)= & (y+x)(u+v)-x u+\left(P_{2}-1\right) y v \\
& -P_{2}(z+y)(v+w)+\left(P_{3}-P_{1} P_{2}+P_{2}\right) z w-W_{1,1}, \\
h_{6}(x, y, z, u, v, w)= & (z+x)(u+w)-x u-\left(P_{1}-1\right) y v \\
& +\left(P_{1}^{2}-P_{2}-P_{1}-1\right) z w+P_{1}(y+z)(v+w)-W_{2,1},
\end{aligned}
$$

then we can show that for $i=0,1,2$, we have

$$
\begin{aligned}
& W_{i, 2 n} \equiv h_{i+1}\left(W_{0, n}, W_{i, n}, W_{2, n}\right), \\
& W_{i, 2 n+1} \equiv h_{i+4}\left(W_{0, n}, W_{i, n}, W_{2, n}, W_{0, n+1}, W_{1, n+1}, W_{2, n+1}\right) \quad(\bmod N) .
\end{aligned}
$$

Also, $W_{0,1} \equiv-2, W_{1,1} \equiv 0, W_{2,1} \equiv Q^{-1}(\bmod N)$, and

$$
W_{i, m n} \equiv W_{i, m}\left(W_{0, n}, W_{1, n}, W_{2, n}, P_{1}, P_{2}, P_{3}\right)(\bmod N) \text {. }
$$

Thus, we can reformulate the final tests (1) and (3) of [14] in a manner analogous to our other reformulations. 
When we implemented these new revisions of tests (1) and (3), using $a=21$, $b=7, h_{i}=1, k_{i}=1, l_{i}=0, G=1$ (for test (1)) and $G=7$ (for test (3)) to determine $P_{1}, P_{2}, P_{3}, Q$ as discussed in [14], we required 39 minutes of AMDAHL CPU time to run each test. Here $\bar{F}_{3}=F_{3} / 7, \bar{F}_{6}=F_{6}$. With $\bar{F}_{1}=F_{1}, S=$ $\bar{F}_{1} \bar{F}_{2} \bar{F}_{3} \bar{F}_{4} \bar{F}_{6}>N^{1 / 3}$, we then ran Lenstra's test as described in Section 2 . As we found no nontrivial factor of $N$, we now know that $N$ must be a prime. This was done in a total of $18+4+16+39+39+6(18 / 60) \approx 118$ minutes of AMDAHL time.

It might be argued here that even though only 2 hours of AMDAHL time was needed to prove $R 1031$ a prime, we still needed in excess of 200 hours of time on the Dubner machine to find the final prime factor $\pi$ which we needed in order to begin our primality proof. In fact, because of the (unexpected) speed of Lenstra's algorithm, we could have proved $R 1031$ a prime without this final factor. Had we used slightly different parameters (select $Q$ in the final tests for the factors of $F_{2}$ such that $(Q / N)=-1$; select $a, b$, for tests (1) and (3) such that $P$ in [14] is not 7) in some of our final tests, we could have put $\bar{F}_{1}=F_{1}, \bar{F}_{2}=F_{2}, \bar{F}_{3}=F_{3}, \bar{F}_{4}=F_{4}, \bar{F}_{6}=F_{6} / \pi$ and have $S=K^{\prime}$, where $K^{\prime}$ is defined in Section 2.

Had these tests been performed, we would know that if $N$ is composite, it must have a prime factor $p$ such that $p \equiv r_{i}(\bmod S), i \in\{1,2,3,4,5,6\}$. But for this value of $S$ we have

$$
N^{1 / 3} / S<31
$$

and $31+S$. Thus, if we use Lenstra's algorithm on the $6 \times 30$ pairs $\left(S^{\prime}, r_{i}^{\prime}\right)$ $(i=1,2,3,4,5,6)$, where $S^{\prime}=31 S, \quad r_{i}^{\prime} \equiv r_{i}(\bmod S)$, and $r_{i}^{\prime} \equiv 1,2,3, \ldots, 30$ $(\bmod 31)$, we could have demonstrated the primality of $N$ in an additional $29 \times 6 \times$ $18 \mathrm{CPU}$ seconds or 53 extra CPU minutes. Nevertheless, we prefer the proof given here as it is more succinct. We do, however, wish to recommend highly the implementation of Lenstra's algorithm in any general or special purpose primality testing routine where its use is relevant. It is very easy to program, executes rapidly, and saves a great deal of time that might otherwise have to be spent on computationally more expensive final tests.

4. Acknowledgment. The authors gratefully acknowledge the enthusiasm and interest displayed in this project by Samuel Yates.

\author{
Department of Computer Science \\ University of Manitoba \\ Winnipeg, Manitoba, Canada R3T 2N2 \\ Dubner Computer Systems, Inc. \\ 158 Linwood Plaza \\ Fort Lee, New Jersey 07024
}

1. L. M. Adelman, C. Pomerance \& R. S. Rumely, "On distinguishing prime numbers from composite numbers," Ann. of Math. (2), v. 117, 1983, pp. 173-206.

2. R. P. Brent \& J. H. Pollard, "Factorization of the eighth Fermat number," Math. Comp., v. 36, 1981, pp. 627-630.

3. J. Brillhart, D. H. Lehmer \& J. L. Selfridge, "New primality criteria and factorizations of $2^{\prime \prime} \pm 1$," Math. Comp., v. 29, 1975, pp. 620-647.

4. J. Brillhart, D. H. Lehmer, John Selfridge, B. Tuckerman \& S. S. Wagstaff, JR., Factorizations of $b^{n}+1, b=2,3,5,6,7,10,11,12$ Up to High Powers, Contemp. Math., vol. 22, Amer. Math. Soc., Providence, R. I., 1983. 
5. H. Cohen \& W. H. Lenstra, JR., “Primality testing and Jacobi sums," Math. Comp., v. 42, 1984, pp. 297-330.

6. H. Cohen \& A. K. Lenstra, “Implementation of a new primality test," Math. Comp. (To appear.)

7. H. Dubner \& R. Dubner, "The development of a powerful, low-cost computer for number theory application," J. Recreational Math. (To appear.)

8. H. W. Lenstra, JR., “Divisors in residue classes," Math. Comp., v. 42, 1984, pp. 331-340.

9. J. M. Pollard, “Theorems on factorization and primality testing," Proc. Cambridge Philos. Soc., v. 76, 1974, pp. 521-528.

10. J. M. Pollard, “A Monte Carlo method for factorization,” BIT, v. 15, 1975, pp. 331-334.

11. H. C. Williams, “A generalization of Lehmer's functions," Acta Arith., v. 29, 1976, pp. 315-341.

12. H. C. Williams, "Factoring on a computer," Math. Intelligencer, v. 6, 1984, pp. 29-36.

13. H. C. Williams \& S. JudD, "Determination of the primality of $N$ by using factors of $N^{2} \pm 1$," Math. Comp., v. 30, 1976, pp. 157-! 72.

14. H. C. Williams \& S. JudD, "Some algorithms for prime testing using generalized Lehmer functions," Math. Comp., v. 30, 1976, pp. 867-886.

15. H. C. Williams \& R. Holte, "Some observations on primality testing," Math. Comp., v. 32, 1978, pp. $905-917$.

16. H. C. Williams \& E. Seah, "Some primes of the form $\left(a^{n}-1\right) /(a-1)$," Math. Comp., v. 33, 1979. pp. 1337-1342. 